\title{
Relationship between genetic mutation variations and acute-phase reactants in the attack-free period of children diagnosed with familial Mediterranean fever
}

\author{
C. Kosan ${ }^{1}$, A. Cayir ${ }^{2}$ and M.I. Turan ${ }^{2}$ \\ ${ }^{1}$ Department of Pediatric Nephrology, Faculty of Medicine, Ataturk University, Erzurum, Turkey \\ ${ }^{2}$ Department of Pediatrics, Faculty of Medicine, Ataturk University, Erzurum, Turkey
}

\begin{abstract}
Familial Mediterranean fever (FMF) is a periodic autoinflammatory disease characterized by chronic inflammation. This study investigated the relationship between acute-phase reactants and gene mutations in attack-free periods of childhood FMF. Patients diagnosed with FMF were divided into four groups based on genetic features: no mutation, homozygous, heterozygous, and compound heterozygous. These groups were monitored for 2 years, and blood samples were collected every 6 months during attack-free periods. Erythrocyte sedimentation rate, C-reactive protein, fibrinogen, and white blood cell count were measured. A disease severity score was determined for each patient. Mean values for erythrocyte sedimentation rate and fibrinogen were significantly different in the homozygous group. White blood cell count and C-reactive protein were similar between the groups. Disease severity score was higher in patients with the M694V mutation than in individuals without the mutation, as well as in those with other mutation groups. Periodic follow-up of patients with FMF MEFV mutations in subjects with acute-phase reactants may be useful in the prevention of morbidity.
\end{abstract}

Key words: Familial Mediterranean fever; Child; Genetic mutation; Acute-phase reactants

\section{Introduction}

Familial Mediterranean fever (FMF) is a recessively inherited, periodic autoinflammatory disease characterized by chronic inflammation (1). The disorder is characterized by recurrent attacks of fever, peritonitis, pleuritis, arthritis, and skin lesions. The typical clinical course is one of exacerbations and remissions. The increased acute-phase response seen during these attacks usually returns to normal in attack-free periods (2,3). Although FMF is a clinical condition generally characterized by painful inflammatory attacks, high levels of acute-phase reactants, cytokines, and inflammation-induced proteins measured during attack-free phases in many patients suggest that inflammation still persists $(4,5)$.

There is no definitive diagnostic laboratory test for FMF. The MEFV gene responsible for the disease has been reported to be on the short arm (16p13.3) of the 16th chromosome. Determination of mutations in the MEFV gene supports diagnosis in patients with clinical findings compatible with FMF. Another finding that supports diagnosis is a rise in acute-phase reactants, known to increase in acute inflammatory events in attack periods. C-reactive protein (CRP), sedimentation, fibrinogen, and white blood cell counts rise during attacks. Serum amyloid-A protein, ceruloplasmin, haptoglobulin, and various cytokines have also been reported to increase during periods of attack (6-9).

Clinical heterogeneity is often observed in FMF, and this is associated with genetic heterogeneity. Several studies have demonstrated that MEFV mutations lead to a nonspecifically increased inflammatory response and that some inflammatory conditions are involved in the etiopathogenesis of the disease. High levels of acute-phase reactants have been measured in asymptomatic individuals with heterozygous MEFV mutations, and inflammation was observed even during the asymptomatic phase in such individuals $(6,10-13)$.

Although search of the literature yielded numerous studies involving acute-phase reactants in FMF patients, 
no long-term study investigating the relationship between gene mutation and acute-phase reactants in attack-free FMF patients was identified.

The present study was intended to determine the values of acute-phase reactants and other laboratory parameters used for routine monitoring, in order to establish the extent to which these are indicative of the patient's clinical status, and to identify any association with FMF gene mutations.

\section{Material and Methods}

This study was performed at the Division of Pediatric Nephrology, Department of Pediatrics, Ataturk University Faculty of Medicine, Turkey. Permission was obtained from parents and adolescent participants. All participants gave written informed consent prior to the study. The study group consisted of 154 ( 74 female and 80 male) ambulatory Turkish children aged 2-18 years who were being monitored for FMF. Diagnosis of FMF was based on laboratory findings and typical clinical features. The same parameters were used for patients evaluated both retrospectively and prospectively.

The study was approved by the local Ethics Committee. We reviewed the records of all patients and recorded the following details: age of onset, genotypes of FMF gene mutations, clinical features (fever and abdominal, thoracic, articular, skin, muscular, testicular, and miscellaneous manifestations), and laboratory parameters including erythrocyte sedimentation rate (ESR), CRP, fibrinogen, and white blood cell count during attack-free periods. Exclusion criteria were the use of any medications known to interfere with hepatic or renal functions and having any cardiac, renal, or hepatic disease at follow-up. The M694V, M680I, V726A, R761H, E148Q, M694I, and P369S MEFV mutations were investigated. The patients were divided into four groups according to genetic features: no mutation, homozygous, heterozygous, and compound homozygous. Homozygous mutation bearing means the same allele being passed on from both mother and father, heterozygous mutations were those identified as occurring in only one area of the exon 10 region, and compound heterozygous mutations were defined as the presence of two or more of the FMF gene mutations in the exon 10 region. Individuals with no mutation comprised a group with clinical FMF diagnostic criteria, despite bearing no mutation, undergoing differential diagnosis for other reasons and with observed response to colchicine. Patients were followed up for 2 years. For each patient, disease severity score was defined according to the criteria previously described by Pras et al. (6).

Patients were recalled after 6 months, and blood samples were collected every 6 months in the 2 years following FMF diagnosis, between 8:00 and 10:00 am after a 12-h fast. All blood samples were obtained in attack-free periods and analyzed immediately. Fasting serum samples were collected during morning hours to avoid diurnal variations. Laboratory parameters included genetic mutation, ESR, CRP, fibrinogen, and white blood cell count.

DNA was extracted from peripheral blood lymphocytes following standard procedures. Mutation identification was performed according to previously described PCR and restriction enzyme digestion or amplification refractory mutation system techniques $(14,15)$.

Multiplex PCR was performed using biotinylated primers for amplification of exons 2, 3, 5, and 10 with a GML SeqFinder Sequencing System MEFV kit (Switzerland). DNA sequencing was conducted on both strands, based on the forward and reverse primers identified in the initial PCR. Next, an ABI 3130 automated DNA sequencer (Applied Biosystems, USA) was used to analyze the strands, and SeqScape v2.6 and Sequencing Analysis 5.3.1 version programs were used to analyze the results.

White blood cell counts were determined using automated equipment (Beckman Coulter LH 750 analyzer, USA). ESR and CRP were determined in whole blood and serum aliquots, respectively. ESR was determined according to the Westergren method and CRP by using a nephelometric method (Beckman Array Protein System, USA). Plasma fibrinogen levels were determined using commercial kits on a STA compact autoanalyzer (STA, France).

\section{Statistical analysis}

All data were subjected to one-way analysis of variance to determine the significance of study parameters between groups of samples. The Tukey post hoc test was used to determine pairwise significance (SSPS 18.0 software, IBM, USA). Differences among groups were obtained using the least significant difference option, and significance was set at $\mathrm{P} \leq 0.05$. Results are reported as means \pm SE.

\section{Results}

One hundred and fifty-four participants with complete data sets were analyzed (74 females, mean age $13.5 \pm 0.4$ years; 80 males, mean age $12.4 \pm 0.4$ years). The genetic features of each group are shown in Table 1.

Comparison of acute-phase reactants including sedimentation and fibrinogen between the groups is shown in Figure 1. A statistically significant difference was found between the groups $(P<0.05)$. However, acute-phase reactants including $\mathrm{CR}$ and white blood cell count were not statistically different between the groups $(P>0.05$; Figure 1).

The correlation between disease severity score and acute-phase reactants including fibrinogen and sedimentation in the study groups is shown in Table 2. 
Table 1. Classification of groups according to genetic mutation.

\begin{tabular}{cccc}
\hline $\begin{array}{c}\text { Group 0 } \\
(\mathrm{n}=18)\end{array}$ & $\begin{array}{c}\text { Group 1 } \\
(\mathrm{n}=33)\end{array}$ & $\begin{array}{c}\text { Group 2 } \\
(\mathrm{n}=51)\end{array}$ & $\begin{array}{c}\text { Group 3 } \\
(\mathrm{n}=52)\end{array}$ \\
\hline- & M694V-M694V & M694V-V726A & M694V \\
M680I-M680I & M694V-M680I & M680I \\
& V726A-V726A & M694V-R761H & V726A \\
& & M694V-E148Q & E148Q \\
& & M694V-M694I & R761H \\
& & M694V-P369S & \\
& & M680I-V726A & \\
& & M680I-R761H & \\
& &
\end{tabular}

Group 0: no mutation; group 1: homozygous; group 2: compound homozygous; group 3: heterozygous.

\section{Discussion}

This study demonstrated significantly higher levels of acute-phase reactants in FMF patients homozygous and heterozygous for M694V mutations compared to those who did not have these mutations. Previous studies have reported a more severe course of disease in patients homozygous for the M694V mutation compared to those without this mutation and have reported a higher risk for developing amyloidosis $(6,10,16)$. Severe clinical symptoms in patients with the M694V mutation may be attributed to increased levels of acute-phase reactants even during the attack-free period. Moreover, on-going inflammation may also be an indicator of imminent complications.

This study identified significantly higher sedimentation and fibrinogen levels during attack-free periods in patients with the M694V mutation than in those without. CRP and white blood cell count did not differ significantly. Variations in acute-phase reactants during an attack and in attackfree periods are used for diagnostic purposes in FMF. Persisting high levels of sedimentation have been reported for some FMF patients between attacks $(13,17)$.

Ergüven et al. (18) reported that fibrinogen levels during periods of attack as well as in attack-free periods were significantly higher in individuals homozygous for the M694V mutation compared to those lacking this mutation. They suggested that this may be associated with the clinically severe course of the disease.

Tunca et al. (13) reported higher CRP and serum amyloid A (SAA) protein levels in patients and in their firstdegree relatives than in the controls. This difference was not noted for other acute-phase reactants. They also reported higher SAA levels in the patient group during the attack-free period, and identified significantly higher SAA levels in individuals homozygous for the M694V mutation. The fact that differences were noted in sedimentation and fibrinogen levels, but not in CRP and white blood cell counts in our study, may be due to obtaining CRP and
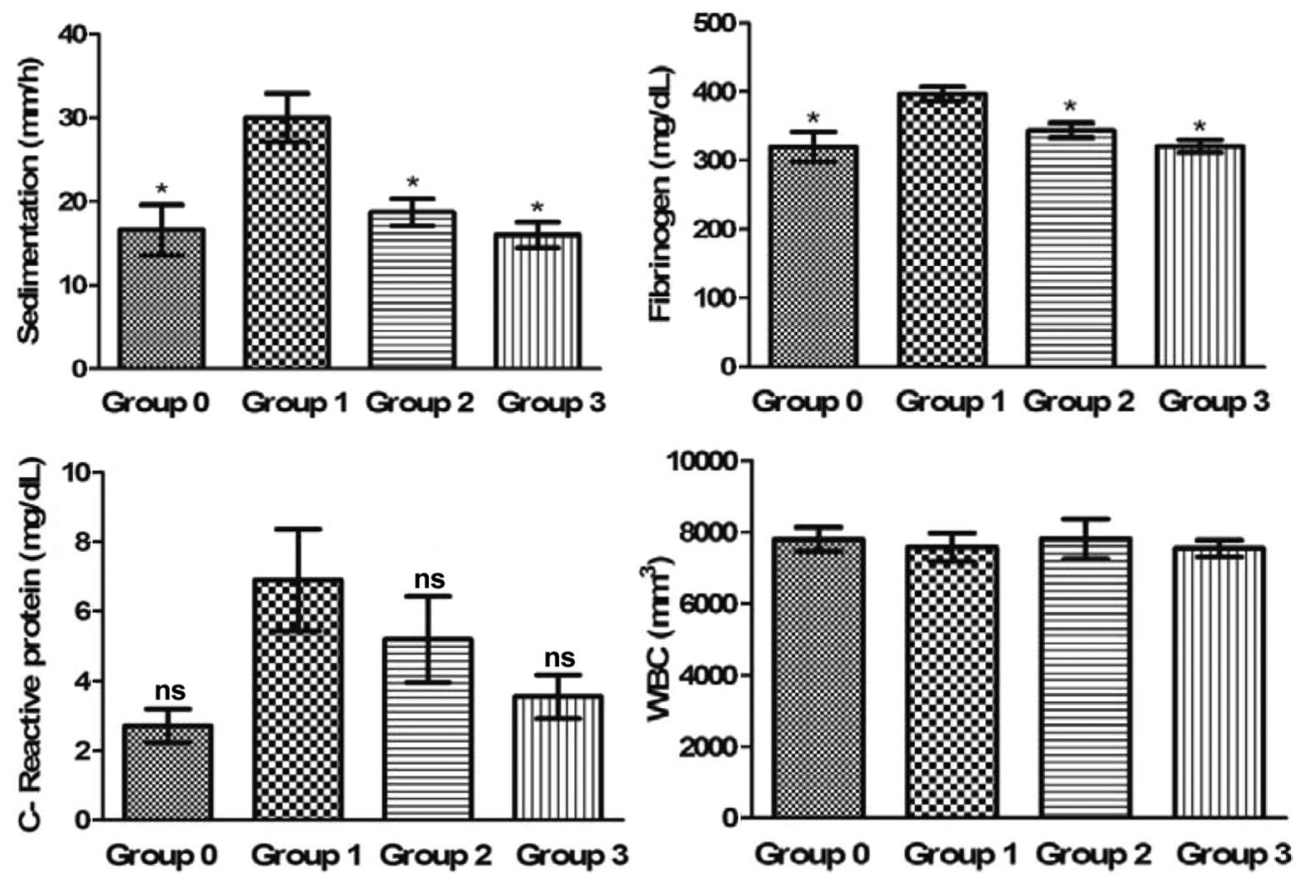

Figure 1. Comparison of the groups in terms of mean measurements of the acute-phase reactants. Blood samples were collected every 6 months during the 2 years following diagnosis of familial Mediterranean fever. Data are reported as means $\pm S E$. Group 0 , no mutation; group 1, homozygous; group 2, compound homozygous; group 3, heterozygous. WBC = white blood cell. One-way analysis of variance and the post hoc Tukey test were used for statistical analyses. ${ }^{*} \mathrm{P} \leq 0.05$, groups 0,2 , and 3 compared to group 1 ; ${ }^{\text {ns }} \mathrm{P}>0.05$, not significant in general, second, third and fourth measurements. 
Table 2. Correlation between disease severity score and acute-phase reactants in study groups.

\begin{tabular}{|c|c|c|c|c|c|c|c|}
\hline Groups & $\begin{array}{l}\text { Disease severity } \\
\text { score }\end{array}$ & Fibrinogen & r & $P$ & Sedimentation & r & $P$ \\
\hline Group 0 & $5.72 \pm 1.1$ & $320 \pm 21.7$ & 0.33 & $<0.05$ & $16.6 \pm 2.98$ & 0.34 & $<0.05$ \\
\hline Group 1 & $7.92 \pm 1.43$ & $397 \pm 10.4$ & & & $30 \pm 2.96$ & & \\
\hline Group 2 & $7.08 \pm 2.23$ & $344 \pm 10.8$ & & & $18.7 \pm 1.62$ & & \\
\hline Group 3 & $7.25 \pm 1.36$ & $321 \pm 9.37$ & & & $16 \pm 1.51$ & & \\
\hline
\end{tabular}

Data are reported as means \pm SE. Group 0: no mutation; group 1: homozygous; group 2: compound homozygous; group 3: heterozygous. The Pearson correlation test was used for statistical analyses.

white blood cell counts at different times than other acutephase reactants. Due to the long half-life of fibrinogen, it is still present at high levels even after the end of inflammation. Because the sedimentation rate of red blood cells is mainly dependent on fibrinogen levels, ESR rises later in inflammation and, likewise, normalizes later. $\mathrm{CRP}$, on the other hand, is apparent 6-10 $\mathrm{h}$ after the first stimulus and reaches maximal levels within $48 \mathrm{~h}$. It soon returns to normal levels (19).

Disease severity score was higher in patients with the M694V mutation than in individuals without the mutation, as well as in those in other mutation groups. This may be a consequence of the inflammatory process persisting during attack-free periods. The disease severity score was positively correlated with both sedimentation and

\section{References}

1. Savic S, Dickie LJ, Battellino M, McDermott MF. Familial Mediterranean fever and related periodic fever syndromes/ autoinflammatory diseases. Curr Opin Rheumatol 2012; 24: 103-112, doi: 10.1097/BOR.0b013e32834dd2d5.

2. Kosan C, Ozkan B. Once-daily use of colchicine in children with familial Mediterranean fever. Clin Pediatr 2004; 43: 605-608, doi: 10.1177/000992280404300703.

3. Fonnesu C, Cerquaglia C, Giovinale M, Curigliano V, Verrecchia E, De Socio G, et al. Familial Mediterranean fever: a review for clinical management. Joint Bone Spine 2009; 76: 227-233, doi: 10.1016/j.jbspin.2008.08.004.

4. Musabak U, Sengul A, Oktenli C, Pay S, Yesilova Z, Kenar L, et al. Does immune activation continue during an attack-free period in familial Mediterranean fever? Clin Exp Immunol 2004; 138: 526-533, doi: 10.1111/j.1365-2249.2004.02632.x.

5. Kosan C, Cayir A, Turan MI, Ustebay S. Paraoxonase 1 and arylesterase levels in children with familial Mediterranean fever. Eur Rev Med Pharmacol Sci 2013; 17: 375-378.

6. Pras E, Langewitz P, Livneh A, Zemer D, Migdal A, Padeh S, et al. Genotype/phenotype correlation in familial Mediterranean fever (a preliminary report). Tel Aviv: Freund Publishing House; 1997.

7. Cazeneuve C, Sarkisian T, Pecheux C, Dervichian M, Nedelec B, Reinert P, et al. MEFV-gene analysis in Armenian patients with familial Mediterranean fever: diagnostic value and unfavorable renal prognosis of the M694V homozygous genotype-genetic and therapeutic fibrinogen. These parameters may be indicators of an occult inflammatory response. The present study demonstrated that the disease burden was high in FMF and that inflammation persisted during symptom-free periods despite regular treatment of patients with the M694V mutation, one of the most important mutations associated with frequent complications.

In conclusion, we believe that monitoring acute-phase reactants (sedimentation rate and fibrinogen levels) at regular intervals during attack-free periods and modifying treatment accordingly, particularly in patients with the M694V mutation, will be beneficial both for the course of the disease and in preventing complications, including amyloidosis and arthritis, associated with increased morbidity.

implications. Am J Hum Genet 1999; 65: 88-97, doi: 10.1086/302459.

8. Tunca M, Akar S, Onen F, Ozdogan H, Kasapcopur O, Yalcinkaya F, et al. Familial Mediterranean fever (FMF) in Turkey: results of a nationwide multicenter study. Medicine 2005; 84: 1-11, doi: 10.1097/01.md.0000152370.84628.0c.

9. Sayhan N, Ozdogan H, Kasapcopur O, Livneh A, van Dijk W. MEFV gene analysis in familial Mediterranean fever patients from Turkey: prognostic 72 value of M694V homozygous phenotype. Clin Exp Rheumatol 2000; 18: 286 (Abstract).

10. Dewalle M, Domingo C, Rozenbaum M, Ben-Chetrit E, Cattan D, Bernot A, et al. Phenotype-genotype correlation in Jewish patients suffering from familial Mediterranean fever (FMF). Eur J Hum Genet 1998; 6: 95-97, doi: 10.1038/ sj.ejhg.5200170.

11. Padeh S, Shinar Y, Pras E, Zemer D, Langevitz P, Pras M, et al. Clinical and diagnostic value of genetic testing in 216 Israeli children with familial Mediterranean fever. J Rheumatol 2003; 30: 185-190.

12. Bakkaloglu A. Familial Mediterranean fever. Pediatr Nephrol 2003; 18: 853-859, doi: 10.1007/s00467-003-1185-2.

13. Tunca M, Kirkali G, Soyturk M, Akar S, Pepys MB, Hawkins PN. Acute phase response and evolution of familial Mediterranean fever. Lancet 1999; 353: 1415, doi: 10.1016/ S0140-6736(99)00990-3.

14. Anonymous. Ancient missense mutations in a new member of the RoRet gene family are likely to cause familial 
Mediterranean fever. The International FMF Consortium. Cell 1997; 90: 797-807, doi: 10.1016/S0092-8674(00)80539-5.

15. French FMF Consortium. A candidate gene for familial Mediterranean fever. Nat Genet 1997; 17: 25-31, doi: 10.1038/ng0997-25.

16. Brik R, Shinawi M, Kepten I, Berant M, Gershoni-Baruch R. Familial Mediterranean fever: clinical and genetic characterization in a mixed pediatric population of Jewish and Arab patients. Pediatrics 1999; 103: e70, doi: 10.1542/peds. 103.5.e70.
17. Drenth PH, Poland D, van het Hof B, Livneh A, van Dijk W. Acute and chronic inflammation in FMF attacks and evidence for acute phase reaction in MEFV heterozygotes. Clin Exp Rheumatol 2000; 18: B-2 (Abstract).

18. Ergüven M, Emeksiz C, Deveci M, Ozlu SG. Relation between microalbuminuria and gene mutations in familia Mediterranean fever. Turk J Pediatr 2008; 50: 326-330.

19. Pincus $\mathrm{M}$, Abraham N. Clinical, laboratory diagnosis and management by laboratory methods. Philadelphia: WB Saunders; 2001. 